\title{
Perfil bioquímico sanguíneo na intoxicação experimental com extrato de Mascagnia rigida (A. Juss.) Griseb. (Malpighiaceae) em coelhos
}

[Biochemistry profile after experimental intoxication by extract of Mascagnia rigida (A. Juss.) Griseb. (Malpighiaceae) in rabbits]

\author{
L.R. Borboleta ${ }^{1}$, C.R. Labarrère ${ }^{1}$, A.F.C. Ribeiro ${ }^{1}$, F.O. Paes-Leme ${ }^{2}$, P.R.O. Paes $^{2}$, \\ N.M. Ocarino ${ }^{2}$, M.M. Melo ${ }^{2 *}$ \\ ${ }^{1}$ Aluna de pós-graduação - EV-UFMG - Belo Horizonte, MG \\ ${ }^{2}$ Escola de Veterinária - UFMG \\ Caixa Postal 567 \\ 30123-970 - Belo Horizonte, MG
}

\begin{abstract}
RESUMO
Avaliou-se o perfil bioquímico sanguíneo na intoxicação por Mascagnia rigida, uma planta tóxica que gera problema econômico para a pecuária, por causar morte súbita. Nove coelhos Nova Zelândia, machos, com massa corporal média de $3,54 \mathrm{~kg}$, foram distribuídos em três grupos $(\mathrm{G})(\mathrm{n}=3)$. Os animais receberam, durante oito dias consecutivos, o equivalente a $30 \mathrm{~g} / \mathrm{kg}$ de matéria seca da planta em dois tipos de extratos: solúvel em água (GS) e insolúvel em água (GI), e formou-se também o grupo-controle (GC). Os exames bioquímicos foram realizados previamente ao início do experimento até o nono dia. A administração dos extratos da Mascagnia rigida causou alterações eletrolíticas que podem justificar alguns sinais clínicos observados e atuar de forma significativa na causa mortis.
\end{abstract}

Palavras-chave: coelho, Mascagnia rigida, planta tóxica, perfil bioquímico

\begin{abstract}
A study was carried out to evaluate the biochemical profile in Mascagnia rigida poisoning, a toxic plant that generates a significant economic problem to livestock, causing "sudden death". Nine New Zealand rabbits, male, $3,54 \mathrm{~kg}$ mean body weight were divided into three groups $(G)(n=3)$. The animals received the equivalent of $30 \mathrm{~g} / \mathrm{kg}$ of dry matter in two types of extracts: water-soluble (GS) and insoluble in water (GI), and the control group (CG) (ultra-pure water) for eight consecutive days. Biochemical exams were done prior to the beginning of the experiment until the ninth day. It was concluded that the administration of extracts of Mascagnia rigida cause electrolyte imbalances that may justify some clinical signs and act significantly in the cause of death.
\end{abstract}

Keywords: rabbit, Mascagnia rigida, toxic plant, biochemical profile

\section{INTRODUÇÃO}

Mascagnia rigida Griseb, da família Malpighiaceae, é uma das plantas tóxicas mais importantes do estado de Minas Gerais, principalmente no norte e nordeste, onde é conhecida popularmente como salsa-rosa e suma-roxa (Melo, 2006).

Recebido em 7 de abril de 2011

Aceito em 5 de agosto de 2011

*Autor para correspondência (corresponding author)

E-mail: mariliamm@ufmg.br
Sob condições naturais, a intoxicação por $M$. rigida acomete principalmente bovinos (Medeiros et al., 2002), sendo descrita também em ovinos e caprinos (Vasconcelos et al., 2008) e jumentos (Silva et al., 2006). Experimentalmente, além das espécies descritas, a planta mostrou-se tóxica para coelhos (Medeiros et al., 2002; Cobucci et al., 2009), ratos (Cunha, 2008) e camundongos (Melo et al., 2008). 
Alguns trabalhos descreveram os sinais clínicos e as alterações histológicas decorrentes da intoxicação por $M$. rigida em diversas espécies, mas poucos estudaram as alterações bioquímicas. Diante de tal contexto, este trabalho teve o objetivo de realizar um estudo dos perfis hepático, renal, cardiomuscular e dos eletrólitos após a administração da Mascagnia rigida utilizando o coelho como modelo experimental.

\section{MATERIAL E MÉTODOS}

Foram utilizados nove coelhos, machos, da raça Nova Zelândia branco, com seis meses de idade e com massa corporal média de $3,54 \mathrm{~kg}$. Inicialmente, os animais foram desverminados (Ivermectina Mectimax 1\% - Agener União Saúde Animal - Brasil.) e alojados em gaiolas metálicas individuais de $90 \times 90 \times 40 \mathrm{~cm}$, respeitando um período de quarentena, recebendo água e ração comercial (Nature Multivita - Socil Evialis $\left.{ }^{\circledR}\right)$ ad libitum.

Para obtenção dos extratos, $12 \mathrm{~kg}$ de folhas maduras de Mascagnia rigida foram colhidas no canteiro de plantas tóxicas da Escola de Veterinária da UFMG, em Belo Horizonte, MG, em julho de 2009. A planta utilizada tem exsicata própria depositada no Herbário de Botânica do Instituto de Ciências Biológicas da UFMG, sob o número BHCB 100819.

As folhas maduras recém-colhidas foram trituradas em liquidificador com água ultrapura, na proporção de $200 \mathrm{~g}$ para $400 \mathrm{~mL}$ de água ultrapura. O material resultante foi colocado em banho ultrassônico por 30 minutos e, após descanso de 10 minutos, foi peneirado, obtendose uma solução aquosa. Esta solução foi mantida em repouso em geladeira por 24 horas, formando duas fases distintas. As fases foram separadas em duas soluções: uma solúvel e outra insolúvel em água, ambas concentradas a vácuo (Dia Pump ${ }^{\circledR}$ Compressor Aspirador Modelo CA) em rotavapor, a $70^{\circ} \mathrm{C}$ em $80 \mathrm{rpm}$.

Para o cálculo do equivalente em matéria seca (MS) e concentração da amostra, foram aliquotadas cinco folhas de $M$. rigida, pesadas e colocadas na estufa em quintuplicata durante 30 minutos. Após a desidratação, foram novamente pesadas, obtendo-se o valor médio de $35 \%$ de MS. As concentrações finais da solução foram de $4,42 \mathrm{~g} / \mathrm{mL}$ de $\mathrm{MS}$ de extrato solúvel e $2,71 \mathrm{~g} / \mathrm{mL}$ de MS de extrato insolúvel de $M$. rigida, com $\mathrm{pH}$ de 5,54 .

Os extratos foram congelados sob temperatura de $-20^{\circ} \mathrm{C}$ em seringas de $20 \mathrm{~mL}$, e três horas antes da administração aos animais, foram retirados do freezer e mantidos em geladeira a $4^{\circ} \mathrm{C}$, após o que foram submetidos ao banho ultrassônico (Branson 1510 - Bransonic ${ }^{\circledR}$ Ultrasonic Cleaner 1510R-MT - USA) por 10 minutos, para homogeneização.

Os coelhos foram distribuídos em três grupos $(n=3)$ e receberam durante oito dias consecutivos, pela via nasoesofágica (Sonda uretral n. 6 em PVC - Markmed $\AA$ ), 30mL de água ultrapura para formar o grupo-controle (GC); ou $26 \mathrm{~mL}$ de extrato aquoso de $M$. rigida solúvel em água ultrapura equivalente a $30 \mathrm{~g} / \mathrm{kg}$ de MS da planta para formar o grupo solúvel (GS); ou $36 \mathrm{~mL}$ de extrato aquoso de $M$. rigida insolúvel em água ultrapura equivalente a $30 \mathrm{~g} / \mathrm{kg}$ de MS da planta para formar o grupo insolúvel (GI). Todos os extratos foram divididos em duas administrações diárias, às $8 \mathrm{~h}$ e $30 \mathrm{~min}$ e às $14 \mathrm{~h}$ e 30min.

Amostras de sangue, $4 \mathrm{~mL}$, foram obtidas da veia marginal da orelha, antes, dia 0 , e após a administração dos extratos, nos dias três, cinco, sete e nove. Cerca de $3 \mathrm{~mL}$ coletados em tubos sem anticoagulante foram submetidos à centrifugação (Centrífuga Excelsa Baby, Fanem, modelo 208N) a 1500G, para separação do soro, o qual foi utilizado para determinações de fosfatase alcalina (FA), alanina aminotransferase (ALT), aspartato aminotransferase (AST), lactato desidrogenase (LDH), creatina quinase (CK), CK fração MB (CK-MB), troponina, ureia, creatinina, magnésio $\left(\mathrm{Mg}^{2+}\right)$, cálcio $\left(\mathrm{Ca}^{2+}\right)$, fósforo $(\mathrm{P})$, cloretos $\left(\mathrm{Cl}^{-}\right)$e glicose por método cinético em aparelho analisador bioquímico (Cobas Mira - Roche - GMI - Global Medical Instrumentations, Inc., Ramsey - EUA). Colheuse $1,0 \mathrm{~mL}$ de sangue em tubo com anticoagulante EDTA, que foi centrifugado a 3.000rpm por 10 minutos para obtenção do plasma, utilizado para determinação da concentração de potássio $\left(\mathrm{K}^{+}\right)$, por meio de leitura em espectrofotômetro (Espectrofotômetro EF1 Metronic Instrumentos Científicos Ltda.) por método colorimétrico (Potássio - Doles Reagentes e Equipamentos para Laboratórios Ltda. - Goiânia - GO). 
O experimento foi realizado em delineamento inteiramente ao acaso, com parcelas subdivididas, sendo as parcelas os grupos, e as subparcelas os tempos. Para avaliação de normalidade e homocedasticidade, foram utilizados os testes de Lilliefors e Bartlett, respectivamente. As variáveis que não apresentaram distribuição normal, tais como CK, CK-MB e CK-MB/CK, sofreram transformação radicial, e outras, como cloretos, creatinina e magnésio, sofreram transformação logarítmica+1. Os dados referentes aos diferentes grupos e tempos foram analisados pelos programas Graphpad Instat ${ }^{\circledR}$ (GraphPad Software, Inc. - Instat - USA) para triagem, SISVAR ${ }^{\circledR}$ (SISVAR ${ }^{\circledR}$ - UFLA - Brasil) para análise de variância $(\mathrm{P}<0,05)$ e análise de regressão $\left(r^{2}>40 \%\right)$. Para os grupos experimentais não foram realizados os testes de hipótese em razão de pequeno grau de liberdade do ensaio.
O projeto de pesquisa foi aprovado pelo CETEA - Comitê de Ética em Experimentação Animal da UFMG (certificado 187/08).

\section{RESULTADOS E DISCUSSÃO}

Durante o período de observação, nenhum coelho dos diferentes grupos experimentais apresentou sinais clínicos de intoxicação. Os valores de FA não apresentaram alteração em relação ao tempo no grupo-controle e nos extratos solúvel e insolúvel. Apesar de os valores de FA (Tab. 1) apresentaram-se abaixo do limite inferior para o coelho proposto por Kaneko et al. (2008), 106,2-133,8U/L, nem todos os intervalos de referência refletem com fidelidade os achados esperados na população de interesse. A não correspondência aos valores de referência tornase irrelevante em razão de os grupos experimentais terem sido constituídos de animais previamente saudáveis e de não terem sido observadas alterações desta enzima nos grupos em relação aos tempos de observação.

Tabela 1. Valores séricos médios de fosfatase alcalina (FA), alanina aminotransferase (ALT) e aspartato aminotransferase (AST) de coelhos submetidos à administração de água ultrapura (controle), extrato solúvel e extrato insolúvel em água de Mascagnia rigida por via nasoesofágica em diferentes tempos

\begin{tabular}{|c|c|c|c|c|}
\hline & $\begin{array}{l}\text { Tempo } \\
\text { (dias) }\end{array}$ & Grupo-controle & Grupo solúvel & Grupo insolúvel \\
\hline \multirow{5}{*}{$\begin{array}{c}\text { FA } \\
(\mathrm{U} / \mathrm{L})\end{array}$} & 0 & $59,33 \pm 21,83$ & $68,33 \pm 20,84$ & $35,33 \pm 8,50$ \\
\hline & 3 & $50,00 \pm 15,59$ & $42,33 \pm 7,57$ & $35,67 \pm 11,59$ \\
\hline & 5 & $45,67 \pm 18,50$ & $46,67 \pm 21,20$ & $46,33 \pm 16,01$ \\
\hline & 7 & $38,67 \pm 13,20$ & $51,67 \pm 15,31$ & $40,33 \pm 15,31$ \\
\hline & 9 & $43,67 \pm 26,50$ & $43,33 \pm 15,50$ & $44,33 \pm 10,07$ \\
\hline \multirow[t]{2}{*}{ Equação } & & $n s$ & ns & $n s$ \\
\hline & 0 & $76,33 \pm 21,73$ & $118,33 \pm 39,93$ & $138,67 \pm 13,8$ \\
\hline \multirow{4}{*}{$\begin{array}{l}\text { ALT } \\
(\mathrm{U} / \mathrm{L})\end{array}$} & 3 & $64,67 \pm 21,55$ & $114,67 \pm 7,02$ & $65,67 \pm 3,79$ \\
\hline & 5 & $113,33 \pm 13,43$ & $96,67 \pm 38,14$ & $138,33 \pm 19,40$ \\
\hline & 7 & $79,33 \pm 5,51$ & $109,67 \pm 35,36$ & $102,67 \pm 40,02$ \\
\hline & 9 & $97,33 \pm 69,40$ & $100,00 \pm 29,14$ & $85,33 \pm 17,50$ \\
\hline \multirow{2}{*}{ Equação } & & & & $y=136,11-52,20$ (dias) \\
\hline & & ns & $n s$ & 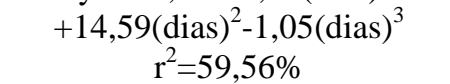 \\
\hline & 0 & $62,00 \pm 26,46$ & $88,00 \pm 21,63$ & $87,67 \pm 16,62$ \\
\hline AST & 3 & $54,00 \pm 34,83$ & $77,00 \pm 2,65$ & $44,00 \pm 13,45$ \\
\hline \multirow[t]{3}{*}{ (U/L) } & 5 & $98,00 \pm 60,63$ & $53,33 \pm 14,84$ & $59,67 \pm 17,79$ \\
\hline & 7 & $78,00 \pm 57,38$ & $60,67 \pm 9,29$ & $45,67 \pm 10,02$ \\
\hline & 9 & $77,00 \pm 43,86$ & $73,67 \pm 30,35$ & $78,00 \pm 33,45$ \\
\hline Equação & & ns & $n s$ & $\begin{array}{c}y=86,28-16,54(\text { dias })+1,71 \text { (dias) })^{2} \\
r^{2}=78,54 \%\end{array}$ \\
\hline
\end{tabular}

Coeficientes de variação: FA - 35,0\%; ALT - 33,6\% e AST- 43,7\%.

$n s$ : não significativo pela análise de variância $(\mathrm{P}>0,05)$. 
Todavia os resultados de FA desta pesquisa diferem dos encontrados por Santos (1975), o qual relatou aumento da FA após a administração de folhas e frutos de $M$. rigida a 12 bovinos durante 30 dias. Como a FA é utilizada como índice colestático e pode indicar obliteração de ductos biliares, o tempo da administração da planta - efeito cumulativo - pode ter sido um fator importante, já que os coelhos receberam $M$. rigida durante oito dias consecutivos.

ALT e AST apresentaram diferença significativa $(\mathrm{P}<0,05)$ em relação ao tempo apenas no grupo insolúvel (Tab. 1). A ALT teve comportamento cúbico, e a AST quadrático. O ponto mínimo calculado para a AST, 46,28U/L, segundo a equação quadrática para $\mathrm{r}^{2}$ de $78,5 \%$, ocorreu no sexto dia. Todos os valores de AST - grupos controle, solúvel e insolúvel nos diferentes tempos - ficaram dentro dos limites estabelecidos por Mader (1997) de 14 a 113U/L. Para os valores de ALT, segundo esse mesmo autor, 48 a 80U/L, podem ser inferidas as mesmas observações feitas em relação à FA.

A enzima citossólica ALT é encontrada em hepatócitos e células musculares esqueléticas dos animais e, como é encontrada em altos níveis em hepatócitos, é utilizada como um marcador usual de injúria hepática. AST é encontrada em diferentes tipos celulares incluindo hepatócitos, eritrócitos e miócitos cardíacos e musculares. Entretanto é mais útil nas avaliações hepatocelular e muscular por causa da sua alta atividade nas células desses tecidos. Como não foi observado aumento de ALT e AST nos coelhos após administração dos extratos de $M$. rigida, pode-se inferir que eles não foram capazes de causar lesão hepática.

Diferentemente deste trabalho, Santos (1975) observou aumento da AST em 12 bovinos que receberam folhas e frutos de $M$. rigida durante 30 dias. Melo et al. (2008) relataram que as frações de $M$. rigida - taninos, flavanoides, alcaloides e saponinas - na dose de $9 \mathrm{~g} / \mathrm{kg}$, $18 \mathrm{~g} / \mathrm{kg}$ e $27 \mathrm{~g} / \mathrm{kg}$, não causaram alteração de ALT em camundongos, entretanto provocaram aumento de AST. Saad et al. (1970), ao estudarem os efeitos tóxicos de uma outra espécie de Mascagnia, M. pubiflora, relataram valores mais elevados tanto de ALT - 66,1U/L como de AST - 119,6 U/L - em cobaias intoxicadas, quando comparados com animais saudáveis, 20,01U/L de ALT, e 26,69U/L de AST. Todavia, Lago (2007) não observou aumento sérico de AST em ovinos que receberam solução aquosa equivalente a $20 \mathrm{~g} / \mathrm{kg}$ de $M$. rigida durante três e sete dias.

A diminuição da concentração plasmática de enzimas se deve a três fatores: diminuição de processo lesional, quando em valores altos; diminuição de sua produção, como em caso de hepatopatias crônicas ou mecanismos inibitórios; e aumento de sua excreção (Stockham e Scott, 2008). Como não ocorreu lesão hepática, suspeita-se que tenha havido inibição da produção dessas enzimas de forma semelhante à descrita por Melo (1998) após intoxicação experimental em caprinos pela Tetrapterys multiglandulosa (Malpighiaceae).

A LDH alterou-se nos grupos solúvel e insolúvel, apresentando comportamento linear no solúvel e quadrático no insolúvel (Tab. 2). O ponto mínimo estimado pela equação no grupo insolúvel, 30,12U/L, ocorreu no quarto dia, para $r^{2}=60,6 \%$. Segundo Mader (1997), as concentrações séricas de LDH no quinto e sétimo dias do GS e no quinto dia do GI estiveram abaixo do valor mínimo de referência para a espécie, 34-129U/L. De forma semelhante ao observado nas enzimas ALT e AST no grupo insolúvel, houve diminuição significativa de LDH nos grupos solúvel e insolúvel.

A LDH é uma enzima presente em vários tecidos, entre eles os de musculatura estriada e esquelética, principalmente em músculos de contração rápida (Campbell, 2004). Todavia, essa enzima, assim como a AST, é pouco específica, tornando necessária a avaliação de outras enzimas, como a CK e a CK-MB, além da troponina, uma subunidade de proteína estrutural muscular (Pinto et al., 2010; Ribeiro et al., 2010) para confirmar lesão muscular. A porcentagem de CK-MB em relação ao valor total de CK fornece um dado confiável quando se avalia uma possível lesão do miocárdio, pois valores aumentados de CK-MB acompanhados de aumento de CK podem ser resultantes de esforço físico, por exemplo. Porém, quando se observa aumento de CK-MB não acompanhado de aumento da CK total, a porcentagem dessa isoenzima fica elevada, comprovando lesão específica do músculo cardíaco (Melo et al., 2008). 
Tabela 2. Valores médios de lactato desidrogenase (LDH) sérica (U/L) de coelhos submetidos à administração de água ultrapura (controle), extrato solúvel e extrato insolúvel em água de Mascagnia rigida por via nasoesofágica em diferentes tempos

\begin{tabular}{ccccc} 
& $\begin{array}{c}\text { Tempo } \\
\text { (dias) }\end{array}$ & Grupo-controle & Grupo solúvel & Grupo insolúvel \\
\hline \multirow{2}{*}{ LDH } & 0 & $57,00 \pm 27,84$ & $89,67 \pm 24,83$ & $63,67 \pm 21,39$ \\
$(\mathrm{U} / \mathrm{L})$ & 3 & $61,67 \pm 22,81$ & $55,00 \pm 1,00$ & $38,70 \pm 13,67$ \\
& 5 & $57,00 \pm 6,00$ & $29,63 \pm 16,00$ & $15,63 \pm 2,49$ \\
& 7 & $43,33 \pm 7,57$ & $24,67 \pm 2,52$ & $52,67 \pm 32,19$ \\
\multirow{2}{*}{ Equação } & 9 & $67,67 \pm 44,46$ & $36,67 \pm 3,21$ & $48,33 \pm 13,01$ \\
& & $n s$ & $\mathrm{y}=78,13-6,46($ dias $)$ & $\mathrm{y}=63,20-13,56($ dias $)+1,39{\text { (dias })^{2}}^{2}$ \\
\hline
\end{tabular}

Coeficiente de variação: LDH - 50,1\%.

ns: não significativo pela análise de variância $(\mathrm{P}>0,05)$.

Não foram observadas alterações $(\mathrm{P}>0,05)$ nos valores séricos médios de CK, CK-MB e \% CKfato justificado pelos altos CV e desvio-padrão MB nos diferentes tempos dos grupos (Tab. 3), observados.

Tabela 3. Valores médios de creatina quinase total (CK) e da fração MB (CK-MB) séricas (U/L) e da porcentagem relativa de CK-MB (\%) de coelhos submetidos à administração de água ultrapura (controle), extrato solúvel e extrato insolúvel em água de Mascagnia rigida por via nasoesofágica em diferentes tempos

\begin{tabular}{c|c|ccc}
\hline & Tempo & & & \\
& (dias) & Grupo-controle & Grupo solúvel & Grupo insolúvel \\
\hline & 0 & $1112,07 \pm 496,21$ & $1006,73 \pm 198,40$ & $577,80 \pm 190,73$ \\
CK & 3 & $928,03 \pm 378,36$ & $778,10 \pm 250,56$ & $669,40 \pm 270,00$ \\
(U/L) & 5 & $1776,80 \pm 577,36$ & $1195,73 \pm 432,18$ & $1021,57 \pm 649,33$ \\
& 7 & $1927,03 \pm 702,35$ & $1559,73 \pm 1111,17$ & $1268,93 \pm 1085,59$ \\
& 9 & $1905,87 \pm 847,49$ & $1165,43 \pm 668,12$ & $616,27 \pm 205,03$ \\
Equação & & $n s$ & $n s$ & $n s$ \\
\hline & 0 & $363,73 \pm 214,02$ & $413,67 \pm 178,01$ & $230,43 \pm 177,92$ \\
CK-MB & 3 & $505,40 \pm 346,66$ & $276,30 \pm 46,55$ & $135,47 \pm 55,25$ \\
(U/L) & 5 & $361,03 \pm 136,12$ & $320,00 \pm 165,85$ & $213,17 \pm 58,56$ \\
& 7 & $317,87 \pm 88,62$ & $224,50 \pm 142,39$ & $287,10 \pm 212,00$ \\
Equação & 9 & $486,27 \pm 142,70$ & $577,70 \pm 578,02$ & $300,30 \pm 116,33$ \\
CK-MB/ & & $n s$ & $n s$ & $n s$ \\
CK (\%) & 3 & $35,89 \pm 27,49$ & $43,68 \pm 26,17$ & $43,33 \pm 29,88$ \\
& 5 & $51,19 \pm 15,02$ & $38,77 \pm 15,38$ & $25,66 \pm 21,92$ \\
& 7 & $20,39 \pm 5,59$ & $25,40 \pm 6,25$ & $24,35 \pm 9,47$ \\
Equação & 9 & $17,20 \pm 4,74$ & $15,06 \pm 4,33$ & $27,44 \pm 14,46$ \\
\hline
\end{tabular}

Coeficientes de variação: CK - 57,4\%; CK-MB - 64,8\%; CK-MB/CK (\%) - 55,7\%.

$n s$ : não significativo pela análise de variância $(\mathrm{P}>0,05)$.

A mensuração da troponina I (cTnI) apresentou resultados negativos em todos os coelhos e em todos os tempos, e o seu uso como marcador específico indica que não houve lesão miocárdica. A concentração normal de cTnI em coelhos sadios varia de 0,012 a $0,014 \mathrm{ng} / \mathrm{dL}$ (Jasinska et al., 2006). Como o teste imunocromatográfico utilizado reage apenas na presença de concentrações acima de $0,5 \mathrm{ng} / \mathrm{mL}$, não é possível descartar a possibilidade de ter havido lesões com liberação de pequena quantidade de cTnI. Porém, lesões cardíacas experimentais em coelhos com o uso desse marcador revelaram aumentos maiores que esse 
limite (Horton et al., 1995), com concentrações plasmáticas de até 2ng/mL (Pinelli et al., 2002).

Os resultados desta pesquisa diferiram dos de Melo et al. (2008), que, após administração das frações de alcaloides e saponinas da $M$. rigida, relataram aumento de $84 \%$ e $70 \%$, respectivamente, de CK-MB em camundongos, concluindo que, especialmente os alcaloides encontrados na $M$. rigida possuem ação tóxica sobre os cardiomiócitos. De forma semelhante, Cobucci et al. (2009) relataram valores de 90,8\% da porcentagem de CK-MB no oitavo dia de administração de $30 \mathrm{~g} / \mathrm{kg}$ do extrato solúvel em água de $M$. rigida a um coelho.
Os resultados da dosagem de ureia e creatinina (Tab. 4) tiveram como objetivo a avaliação da função renal. A administração dos extratos solúvel e insolúvel de $M$. rigida não causou alteração na função renal dos animais nos tempos estudados $(\mathrm{P}>0,05)$, conforme também relatado por Lago (2007), após administrar $20 \mathrm{~g} / \mathrm{kg}$ de suspensão aquosa de $M$. rigida para ovinos durante três e sete dias consecutivos. De acordo com valores de referência de Mader (1997), 13 a $29 \mathrm{mg} / \mathrm{dL}$ de ureia e 0,5 a $2,5 \mathrm{mg} / \mathrm{dL}$ de creatinina não resultaram em azotemia nos animais estudados.

Tabela 4. Valores médios de ureia e creatinina séricas de coelhos submetidos à administração de água ultrapura (controle), extrato solúvel e extrato insolúvel em água de Mascagnia rigida por via nasoesofágica em diferentes tempos

\begin{tabular}{ccccc}
\hline & $\begin{array}{c}\text { Tempo } \\
\text { (dias) }\end{array}$ & Grupo-controle & Grupo solúvel & Grupo insolúvel \\
\hline Ureia & 0 & $18,67 \pm 2,89$ & $22,33 \pm 2,31$ & $21,00 \pm 2,00$ \\
$(\mathrm{mg} / \mathrm{dL})$ & 3 & $21,00 \pm 6,56$ & $18,33 \pm 1,15$ & $19,00 \pm 1,00$ \\
& 5 & $19,67 \pm 2,31$ & $18,67 \pm 3,51$ & $17,00 \pm 2,65$ \\
& 7 & $18,67 \pm 2,52$ & $22,00 \pm 4,36$ & $21,00 \pm 1,00$ \\
Equação & 9 & $18,33 \pm 3,06$ & $20,67 \pm 5,51$ & $20,33 \pm 4,16$ \\
Creatinina & & $n s$ & $n s$ & $n s$ \\
(mg/dL) & 3 & $1,17 \pm 0,99$ & $1,00 \pm 0,10$ & $0,97 \pm 0,06$ \\
& 5 & $0,90 \pm 0,10$ & $1,07 \pm 0,15$ & $1,00 \pm 0,10$ \\
Equação & 7 & $1,27 \pm 0,35$ & $1,27 \pm 0,40$ & $0,93 \pm 0,31$ \\
& 9 & $0,87 \pm 0,81$ & $0,83 \pm 0,15$ & $0,87 \pm 0,12$ \\
\end{tabular}

Coeficientes de variação: Ureia - 16,0\%; Creatinina - 37,3\%.

ns: não significativo à transformação quadrática (creatinina), pela análise de variância $(\mathrm{P}>0,05)$.

Alguns trabalhos relataram degeneração hidrópico-vacuolar dos túbulos contorcidos distais como a principal lesão causada pela $M$. rigida (Gava et al., 1998; Vasconcelos et al., 2008), porém sem acompanhamento de urinálise e avaliação de provas bioquímicas da função renal. Portanto, não existe paralelo entre degeneração hidrópico-vacuolar dos túbulos contorcidos distais e da função renal. Segundo Campbell (2004), é necessária a perda de 50 a $75 \%$ da função renal para o aparecimento de azotemia e, para isso, a classificação da alteração morfológica teria que ser de moderada a intensa.

Os trabalhos de autores que relataram degeneração hidrópico-vacuolar em animais intoxicados por $M$. rigida não classificaram o grau de acometimento histopatológico. Porém, como foi descrito polaciúria em animais intoxicados por $M$. rigida (Santos, 1975; Lago, 2007), a degeneração dos túbulos contorcidos distais poderia levar a esse sinal clínico, uma vez que essa estrutura renal em sua parte final é responsável pela reabsorção de água e sódio e secreção de potássio (Guyton e Hall, 2006).

Lago (2007) não encontrou alteração histológica nos rins de ovinos intoxicados com $M$. rigida que indicasse comprometimento de função e sugeriu que a polaciúria também observada por Santos (1975) em bovinos intoxicados por folhas e frutos de $M$. rigida possa ter ocorrido em razão do aumento da taxa de filtração glomerular causada pelo aumento da frequência cardíaca. Portanto, pode-se observar que, pela avaliação dos perfis hepático, renal e cardiomuscular, os 
extratos - solúvel e insolúvel em água - de folhas de $M$. rigida não causaram alterações que comprometessem a função desses órgãos.

Em relação aos eletrólitos, não foi observada diferença estatística $(\mathrm{P}>0,05)$ na mensuração sérica de potássio (Tab. 5). Segundo Kaneko et al. (2008), os valores de potássio, 4,8 a $5,8 \mathrm{mmol} / \mathrm{L}$, apresentaram discreta diminuição no grupo controle, no quinto dia, $4,34 \mathrm{mmol} / \mathrm{L}$, e no grupo insolúvel, nos sétimo e nono dias, 4,27 e 4,32mmol/L, respectivamente. De acordo com a classificação de Mader (1997), os valores encontrados neste estudo estão dentro dos limites normais de 3,6 a $6,9 \mathrm{mg} / \mathrm{dL}$.

Em relação ao magnésio, houve alteração $(\mathrm{P}>0,05)$ nos tempos dos grupos estudados. Essa variável teve comportamento quadrático no GI $\left(\mathrm{r}^{2}=43,3 \%\right)$ com ponto mínimo ocorrendo no quinto dia, enquanto no GC e no GS ocorreu melhor ajustamento ao modelo cúbico, $\mathrm{r}^{2}=41,4 \%$ e 60,3\%, respectivamente. Segundo Kaneko et al. (2008), os valores séricos de magnésio deste trabalho, 2,09 a 2,41mg/dL, apresentaram-se diminuídos no quinto dia dos grupos GS e GI, e no sétimo dia do GC.

Tabela 5. Valores médios de potássio plasmático e magnésio sérico de coelhos submetidos à administração de água ultrapura (controle), extrato solúvel e extrato insolúvel em água de Mascagnia rigida por via nasoesofágica em diferentes tempos

\begin{tabular}{|c|c|c|c|c|}
\hline & Tempo (dias) & Grupo controle & Grupo solúvel & Grupo insolúvel \\
\hline \multirow{5}{*}{$\begin{array}{l}\text { Potássio } \\
\text { (mmol/L) }\end{array}$} & 0 & $5,34 \pm 0,74$ & $5,76 \pm 0,78$ & $4,86 \pm 1,31$ \\
\hline & 3 & $5,05 \pm 1,16$ & $4,77 \pm 0,76$ & $4,92 \pm 0,74$ \\
\hline & 5 & $4,34 \pm 0,42$ & $5,24 \pm 0,53$ & $5,03 \pm 1,46$ \\
\hline & 7 & $4,94 \pm 0,32$ & $5,89 \pm 1,74$ & $4,27 \pm 0,21$ \\
\hline & 9 & $4,88 \pm 0,20$ & $4,82 \pm 1,26$ & $4,32 \pm 0,23$ \\
\hline Equação & & $n s$ & $n s$ & $n s$ \\
\hline \multirow{5}{*}{$\begin{array}{c}\text { Magnésio } \\
\text { (mg/dL) }\end{array}$} & 0 & $2,13 \pm 0,21$ & $2,50 \pm 0,44$ & $2,27 \pm 0,21$ \\
\hline & 3 & $2,27 \pm 0,31$ & $2,60, \pm 0,40$ & $2,23 \pm 0,15$ \\
\hline & 5 & $2,47 \pm 0,31$ & $1,87 \pm 0,42$ & $1,47 \pm 0,06$ \\
\hline & 7 & $1,90 \pm 0,10$ & $2,23 \pm 0,23$ & $2,23 \pm 0,15$ \\
\hline & 9 & $2,17 \pm 0,32$ & $2,47 \pm 0,23$ & $2,40 \pm 0,36$ \\
\hline Equação & & $\begin{array}{c}\mathrm{y}=2,11+0,27 \text { (dias) } \\
-0,07(\text { dias })^{2}+0,004(\text { dias })^{3} \\
r^{2}=41,04 \%\end{array}$ & $\begin{array}{c}\mathrm{y}=2,52+0,16(\text { dias }) \\
-0,08(\text { dias })^{2}+0,007(\text { dias })^{3} \\
r^{2}=60,27 \%\end{array}$ & $\begin{array}{c}\mathrm{y}=2,33-0,21 \text { (dias) } \\
+0,02(\text { dias })^{2} \\
\mathrm{r}^{2}=43,28 \%\end{array}$ \\
\hline
\end{tabular}

Coeficientes de variação: potássio - 17,9\%; magnésio - 16,6\%.

ns: não significativo pela análise de variância $(\mathrm{P}>0,05)$.

A hipocalemia ocorre quando há saída do potássio do compartimento extracelular para o intracelular, diminuição do consumo dietético ou aumento da perda de potássio pelos rins, trato digestivo ou pele. As causas renais, poliúria e aumento do fluxo tubular renal, podem promover maior excreção de potássio (Stockham e Scott, 2008). Neste estudo, não houve alteração da dieta dos animais em nenhum momento e tampouco foi observada poliúria. Já a hipercalemia resultante de miotoxicicade poderia exercer um efeito disrritmogênico (prolongação de Q-T e alta amplitude) sobre o coração (Kaneko et al., 2008). Por ser intracelular, as lesões celulares causariam aumento nas concentrações séricas desse eletrólito (Carlson,
1997), o que também não foi observado neste estudo.

Pereira et al. (1996) não encontraram alterações séricas do magnésio em caprinos experimentalmente intoxicados com folhas verdes e secas de $M$. rigida (5 a $20 \mathrm{~g} / \mathrm{kg}$ ). O magnésio é um importante regulador de múltiplos processos cardiovasculares, incluindo a condução elétrica do miocárdio e a contratilidade, o fluxo transmembrana de cálcio, o transporte de potássio, o tônus do músculo liso vascular, a reatividade coronariana e a síntese de óxido nítrico (Noronha e Matuschak, 2002). Na medida em que o magnésio é um cofator essencial para a manutenção do potencial 
transmembrana do miocárdio, a hipomagnesemia diminui o limiar para arritmias (Booth et al., 2003). Os efeitos tóxicos de $M$. rigida na fibra cardíaca podem estar associados com a capacidade ou não dessa planta em causar hipomagnesemia, que poderia predispor ao aparecimento de arritmias e ser uma possível causa de morte súbita na intoxicação.

A concentração sérica de cálcio diminuiu significativamente $(\mathrm{P}<0,05)$ no GS (Tab. 6). A variável apresentou comportamento quadrático, com $\mathrm{r}^{2}$ de $71,2 \%$ e ponto mínimo ocorrendo no sexto dia, $12,48 \mathrm{mg} / \mathrm{dL}$. Os valores de cálcio mantiveram-se dentro dos valores de referência para a espécie propostos por Kaneko et al. (2008), de 5,8 a 14,0mg/dL. O cálcio participa da contração cardíaca e da condução do estímulo elétrico ventricular, sendo um íon importante da fase de platô (fase 2), que corresponde ao intervalo Q-T (Lago et al., 2009). Havendo distúrbio de cálcio, o ECG mostrará intervalo QT curto na hipercalcemia e intervalo QT longo na hipocalemia (Ramos e Souza, 2007). O cálcio também mantém uma relação de homeostase com o fósforo: o aumento de fósforo pode alterar o cálcio ionizado na medida em que aumenta o cálcio ligado a este íon e diminui o cálcio ionizado, importante para as contrações muscular esquelética e cardíaca.

Os animais dos grupos GC e GI apresentaram alterações $(\mathrm{P}<0,05)$ do fósforo ao longo do tempo, entretanto não se observou ajuste do GC aos modelos de regressão propostos (Tab. 6). No GI, seu comportamento foi linear ao longo do tempo, com $\mathrm{r}^{2}$ de 81,4\%. Ocorreu discreta hipofosfatemia a partir do quinto dia, segundo Mader (1997), 4,0 a 6,9mg/dL, e Kaneko et al. (2008), 3,70 a 4,62mg/dL.

Tabela 6. Valores médios de cálcio e fósforo séricas de coelhos submetidos à administração de água ultrapura (controle), extrato solúvel e extrato insolúvel em água de Mascagnia rigida por via nasoesofágica em diferentes tempos

\begin{tabular}{|c|c|c|c|c|}
\hline & $\begin{array}{l}\text { Tempo } \\
\text { (dias) }\end{array}$ & Grupo-controle & Grupo solúvel & Grupo insolúvel \\
\hline \multirow{5}{*}{$\begin{array}{l}\text { Cálcio } \\
\text { (mg/dL) }\end{array}$} & 0 & $13,73 \pm 0,83$ & $14,83 \pm 1,40$ & $12,80 \pm 0,62$ \\
\hline & 3 & $13,80 \pm 0,61$ & $12,93 \pm 0,40$ & $12,97 \pm 0,90$ \\
\hline & 5 & $14,27 \pm 0,60$ & $12,30 \pm 0,10$ & $12,30 \pm 1,11$ \\
\hline & 7 & $12,83 \pm 0,95$ & $13,70 \pm 0,52$ & $13,20 \pm 1,15$ \\
\hline & 9 & $13,43 \pm 0,81$ & $13,20 \pm 0,53$ & $13,40 \pm 0,96$ \\
\hline Equação & & ns & $\begin{array}{c}\mathrm{y}=14,71-0,73(\text { dias })+0,06(\text { dias })^{2} \\
\mathrm{r}^{2}=71,16 \%\end{array}$ & ns \\
\hline \multirow{5}{*}{$\begin{array}{l}\text { Fósforo } \\
\text { (mg/dL) }\end{array}$} & 0 & $4,93 \pm 0,32$ & $4,30 \pm 0,95$ & $5,13 \pm 0,32$ \\
\hline & 3 & $4,87 \pm 0,42$ & $4,60 \pm 2,00$ & $4,73 \pm 0,35$ \\
\hline & 5 & $5,60 \pm 0,70$ & $4,70 \pm 0,53$ & $3,67 \pm 0,72$ \\
\hline & 7 & $3,87 \pm 0,71$ & $4,77 \pm 0,31$ & $3,53 \pm 0,06$ \\
\hline & 9 & $4,40 \pm 0,56$ & $4,70 \pm 0,17$ & $3,67 \pm 0,31$ \\
\hline $\begin{array}{l}\text { Equação } \\
\text { P(mg/dL) }\end{array}$ & & $\begin{array}{c}y=5,16-0,08 \text { (dias) } \\
r^{2}=23,03 \%\end{array}$ & $n s$ & $\begin{array}{c}y=5,05-0,19 \text { (dias) } \\
r^{2}=81,43 \%\end{array}$ \\
\hline
\end{tabular}

Coeficientes de variação: cálcio - 7,2\% e fósforo - 18,4\%.

ns: não significativo pela análise de variância $(\mathrm{P}>0,05)$.

Os resultados desta pesquisa demonstraram diminuição de magnésio, cálcio e fósforo após administração do extrato aquoso de $M$. rigida para coelhos, diferindo dos resultados de Santos (1975), que relatou não variação nos valores de fósforo e cálcio de bovinos intoxicados por folhas e frutos de $M$. rigida $(4 \mathrm{~g} / \mathrm{kg})$ durante 30 dias. Todavia, Pereira et al. (1996) observaram que 5 a $20 \mathrm{~g} / \mathrm{kg}$ de folhas verdes e secas de $M$. rigida causaram alterações nas concentrações séricas de potássio, fósforo e magnésio em caprinos.

A mensuração de cloretos apresentou, no grupo insolúvel, seu ponto máximo no sexto dia, 131,33mEq/L (Tab. 7), segundo a regressão quadrática e $\mathrm{r}^{2}$ de 67,2\% (P>0,05). De acordo com Campbell (2004), ocorreu hipercloremia no 
grupo extrato insolúvel aos três, cinco, sete e nove dias após, 89 - 120mEq/L. Podem ser citadas afecções que cursam com hipercloremia, como a insuficiência renal aguda, acidose metabólica, síndrome nefrótica, diabetes insipidus, na hiperfunção adrenocortical e hiperparatireoidismo (Stockham e Scott, 2008), que também aumentariam os níveis de cálcio. Insuficiência renal deve ser descartada neste estudo pela avaliação da função renal que se apresentou normal e diabetes insipidus, pela ausência de poliúria.

Tabela 7. Valores médios de cloretos séricos $(\mathrm{mEq} / \mathrm{l})$ de coelhos submetidos à administração de água ultrapura (controle), extrato solúvel e extrato insolúvel em água de Mascagnia rigida por via nasoesofágica em diferentes tempos

\begin{tabular}{cccc}
\hline \multirow{2}{*}{$\begin{array}{c}\text { Tempo } \\
\text { (dias) }\end{array}$} & Grupo-controle & Grupo solúvel & \\
\cline { 2 - 4 } & $110,00 \pm 6,00$ & $116,67 \pm 8,62$ & Grupo insolúvel \\
\hline 0 & $112,33 \pm 7,64$ & $116,00 \pm 17,35$ & $120,67 \pm 3,06$ \\
5 & $111,67 \pm 4,73$ & $114,67 \pm 16,26$ & $130,67 \pm 1,53$ \\
7 & $110,33 \pm 9,45$ & $113,33 \pm 9,45$ & $133,00 \pm 3,00$ \\
9 & $107,00 \pm 0,00$ & $111,00 \pm 16,46$ & $126,33 \pm 3,51$ \\
Equação & $n s$ & $n s$ & $130,00 \pm 5,57$ \\
& & & $y=121,47+3,47($ dias $)$ \\
& & & $-0,305(\text { dias })^{2}$ \\
& & & $\mathrm{r}^{2}=67,21 \%$ \\
\hline
\end{tabular}

Coeficiente de variação: $\mathrm{Cl}^{-}-$9,56\%.

ns: não significativo pela análise de variância $(\mathrm{P}>0,05)$.

As concentrações séricas de glicose também não apresentaram alterações em relação aos tempos estudados (Tab. 8) e estão dentro dos limites de referência para a espécie de75 a 155mg/dL (Mader, 1997). Pode-se afirmar que não houve estresse, que resultaria em hiperglicemia pela liberação de glicocorticoides e catecolaminas (Stockham e Scott, 2008) ou hipoglicemia devido ao jejum prolongado, hiporexia/anorexia ou má absorção provocada pelos constituintes tóxicos da Mascagnia rigida e sua manifestação clínica.

Tabela 8. Valores médios de glicose sérica (mg/dL) de coelhos submetidos à administração de água ultrapura (controle), extrato solúvel e extrato insolúvel em água de Mascagnia rigida por via nasoesofágica em diferentes tempos

\begin{tabular}{cccc}
\hline Tempo & \multicolumn{3}{c}{ Glicose $(\mathrm{mg} / \mathrm{dL})$} \\
\cline { 2 - 4 } (dias) & Grupo-controle & Grupo solúvel & Grupo insolúvel \\
\hline T zero & $111,00 \pm 17,35$ & $118,33 \pm 11,93$ & $110,00 \pm 3,46$ \\
T1 & $102,67 \pm 14,36$ & $121,33 \pm 8,62$ & $122,67 \pm 10,07$ \\
T2 & $131,67 \pm 17,21$ & $118,00 \pm 10,39$ & $114,67 \pm 7,02$ \\
T3 & $112,00 \pm 14,73$ & $119,33 \pm 1,53$ & $116,00 \pm 1,00$ \\
T4 & $123,67 \pm 22,68$ & $115,33 \pm 7,02$ & $108,00 \pm 2,65$ \\
Equação & $n s$ & $n s$ & $n s$ \\
\hline
\end{tabular}

Coeficiente de variação: Glicose - 10,3\%.

ns: não significativo pela análise de variância $(\mathrm{P}>0,05)$.

Fica evidente que tanto o extrato solúvel como o extrato insolúvel de $M$. rigida possuem propriedades capazes de causar alterações eletrolíticas. Uma das hipóteses é que a alteração eletrolítica, como um dos efeitos tóxicos da $M$. rigida, poderia desestabilizar o potencial de repouso das membranas celulares e afetar várias vias metabólicas dependentes desses íons. Sendo o coração um órgão dependente do equilíbrio hidroeletrolítico para formação e condução do impulso elétrico, denota-se um possível potencial cardiotóxico da planta. Alguns autores sugerem que a arritmia cardíaca seja uma das possíveis causas de morte súbita na intoxicação por $M$. rigida, todavia isso ainda não foi comprovado experimentalmente. Os efeitos cumulativos da $M$. rigida e os mecanismos que contribuem para a morte súbita, contudo, não foram estudados. 


\section{CONCLUSÕES}

Não houve alteração das funções hepática, cardiomuscular e renal de coelhos experimentalmente intoxicados com os extratos aquosos de $M$. rigida. Entretanto, ocorreram alterações no magnésio, cálcio, fósforo e cloretos, sugerindo que a intoxicação por $M$. rigida pode, também, ser decorrente de alterações eletrolíticas.

\section{REFERÊNCIAS BIBLIOGRÁFICAS}

BOOTH, J.V.; PHILLIPS-BUTE, B.; McCANTS, C.B et al. Low serum magnesium level predicts major adverse cardiac events after coronary artery bypass graft surgery. Am. Heart J., v.145, p.1108-1113, 2003.

CAMPBELL, T.W. Hematologia de mamíferos: Animais de laboratório e espécies variadas. In: THRALL, M.A.; BAKER, D.C.; CAMPBELL, T.W et al. (Eds.). Hematologia e bioquímica clínica veterinária. São Paulo: Roca, 2004. 582p.

CARLSON, G.P. Fluid, eletrolyte and acid-base balance. In: KANEKO, J.J.; HARVEY, J.W.; BRUSS, M.L. (Eds.). Clinical biochemistry of domestic animals. 5.ed. San Diego: Academic, 1997. p.485-516.

COBUCCI, G.C.; MELO, M.M.; LABARRÈRE, C.R et al. Disfunção cardíaca na intoxicação experimental com extrato aquoso de Mascagnia rigida. In: SEMANA DE INICIAÇÃO CIENTÍFICA DA UFMG, 18., 2009, Belo Horizonte. Anais... Belo Horizonte: UFMG, 2009.

CUNHA, L.C. Avaliação dos efeitos tóxicos da Mascagnia rigida em ratos: estudo anatomopatológico; comparação entre metodologias cromatográficas para detecção do fluoracetato de sódio. 2008. 100f. Dissertação (Mestrado em Medicina Veterinária) - Faculdade de Medicina Veterinária e Zootecnia, Universidade de São Paulo, São Paulo, SP.

GAVA, A.; CRISTANI, J.; BRANCO, J.V. et al. Mortes súbitas em bovinos causadas pela ingestão de Mascagnia sp. (Malpighiaceae) no Estado de Santa Catarina. Pesqui. Vet. Bras., v.18, p.16-20, 1998.
GUYTON, A.C.; HALL, J.E. (Eds.). Urine formation by the kidneys: II. Tubular processing of the glomerular filtrate. In:__ Textbook of medical physiology. 11.ed. Philadelphia: Elsevier Saunders, 2006. p.336.

HORTON, J.W.; GARCIA, N.M.; WHITE, D.J. et al. Postburn cardiac contractile function and biochemical markers of postburn cardiac injury. J. Am. Coll. Surg., v.181, p.289-298, 1995.

JASINSKA, M.; OWCZAREK, J.; ORSZULAKMICHALAK, D. The influence of simvastatin at high dose and diltiazem on myocardium in rabbits, the biochemical study. Acta Pol. Pharm., v.63, p.386-390, 2006.

KANEKO, J.J.; HARVEY, J.W.; BRUSS, M.L. (Eds). Blood analyte reference values in some laboratory animals. In:__. Clinical biochemistry of domestic animal. 5.ed. Philadelphia: Academic, 2008. Appendix IX, p. 881-887.

LAGO, E.P. Intoxicação experimental em ovinos por Mascagnia rigida (A. Juss.) Griseb. (Malpighiaceae): estudo fitoquímico, fitoanatômico e aspectos clínicos, laboratoriais e ecocardiográficos. 2007. 65f. Tese (Doutorado em Medicina Veterinária) - Escola de Veterinária, Universidade Federal de Minas Gerais, Belo Horizonte, MG.

LAGO, E.P.; MELO, M.M.; ARAÚJO, R.B. et al. Perfis eletrocardiográfico e ecodopplercardiográfico de ovinos após ingestão da suspensão aquosa de Mascagnia rigida Griseb. (Malpighiaceae). Arq. Bras. Med. Vet. Zootec., v.61, p.853-862, 2009.

MADER, D.R. Rabbits: basic approach to veterinary care. In: HILLYER, E.V.; QUESENBERRY, K.E. (Eds.). Ferrets, rabbits, and rodents: clinical medicine and surgery. Philadelphia: W.B. Saunders, 1997. p.160-168.

MEDEIROS, R.M.T.; NETO, S.A.G.; BARBOSA, R.C. et al. Sudden bovine death from Mascagnia rigida in Northeastern Brazil. Vet. Hum. Toxicol., v.44, p.286-288, 2002.

MELO, M. M. Estudo fitoquímico e intoxicação experimental pela Tetrapterys multiglandulosa $A$. JUSS. (Malpighiaceae) em cabras gestantes. 1998. 306f. Tese (Doutorado em Medicina Veterinária) - Escola de Veterinária, Universidade Federal de Minas Gerais, Belo Horizonte, MG. 
MELO, M.M. Plantas que causam intoxicação aguda. Cad. Tec. Vet. Zootec., n.49, p.4-9, 2006.

MELO, M.M.; VERÇOSA JÚNIOR, D.; PINTO, M.C.L. et al. Intoxicação experimental com extratos de Mascagnia rigida (Malpighiaceae) em camundongos. Arq. Bras. Med. Vet. Zootec., v.60, p.631-640, 2008.

NORONHA, J.L.; MATUSCHAK, G.M. Magnesium in critical illness: metabolism, assessment, and treatment. Intensive Care Med., v.28, p.667-679, 2002.

PEREIRA, A.S.; SANTOS, L.F.L.; NUNESPINHEIRO, D.C.S. Alterações bioquímicas e patológicas em caprinos intoxicados por tinguí (Mascagnia rigida). In: CONGRESSO PANAMERICANO DE CIÊNCIAS VETERINÁRIAS. 15., 1996, Campo Grande, MS. Anais... Campo Grande: Associação Panamericana de Ciências Veterinárias, 1996. v.1, p.152.

PINELLI, A.; TRIVULZIO, S.; TOMASONI, L. et al. Cardiac necrosis markers associated with low nitric oxide levels in the plasma of rabbits after treatment with vasopressin: protective effects of nitroglycerin administration. Pharmacol. Res., v.45, p.427-434, 2002.

PINTO M.C.L.; MELO M.B.; CRUZ M.L. et al. Cardiorespiratory evaluation of juvenile rats experimentally envenomed with Tityus serrulatus venom. J. Venom Anim. Toxins incl. Trop. Dis., v.16, p.253-267, 2010.

RAMOS, A.P.; SOUZA, B.S. Eletrocardiograma: princípios, conceitos e aplicações. [s.l.]: Centro de Estudos de Fisiologia do Exercício - CEFE, 2007. 15p.
RIBEIRO, E. L.; PINTO, M.C.L.; LABARRÈRE, C.R. et al. Biochemical profile of dogs experimentally envenomed with Tityus serrulatus scorpion venom. Toxicon, v.55, p.1125-1131, 2010.

SAAD, A.D.; ANDRADE, S.O.; AGUIAR, A.A. The toxic effects of Mascagnia publiflora (Juss.) Griseb. An. Acad. Bras. Cienc., v.42, p.235-244, 1970.

SANTOS, H.L. Aspectos clínicos, laboratoriais e anatomo-histopatológicos na intoxicação experimental de bovinos pela Mascagnia rigida.1975. 36f. Tese (Mestrado) - Escola de Veterinária, Universidade Federal de Minas Gerais, Belo Horizonte, MG.

SILVA, D.M.; RIET-CORREA, F.; MEDEIROS, R.M.T. et al. Plantas tóxicas para ruminantes e equídeos no Seridó Ocidental e Oriental do Rio Grande do Norte. Pesqui. Vet. Bras., v.26, p.223236, 2006.

STOCKHAM, S.L.; SCOTT, M.A. (Eds.). Introductory concepts. In:__. Fundamentals of veterinary clinical pathology. 2.ed. Iowa: Blackwell, 2008. p.20.

VASCONCELOS, J.S.; RIET-CORREA, F.; DANTAS, A.F.M. et al. Intoxicação por Mascagnia rigida (Malpighiaceae) em ovinos e caprinos. Pesqui. Vet. Bras., v.28, p.521-526, 2008. 